\title{
Ueber die japanischen Gallen.
}

Von C. Hartwich in Tangermünde.

Meiner "Uebersicht der technisch und pharmaceutisch verwendeten Gallen" (d. Z. 1883. Bd. 221, S. 881 u. f.) habe ich bezüglich der oben genannten Gallen einige Bemerkungen nachzutragen: Durch die Güte des Herrn von Schlechtendahl in Halle gelangte ich in den Besitz einer Anzahl von demselben in chinesischen Gallen gefundener geflügelter Insekten (derselben, nach denen Lichtenstein das Genus Schlechtendalia aufstellte (meine Uebersicht pag. 894); da ich gleichzeitig in einigen japanischen Gallen geflügelte Insekten gefunden, war ich in den Stand gesetzt, beide vergleichen zu können, und es stellte sich heraus, dass die Insekten beider Gallen durchaus identisch sind, dass also auch die japanischen Gallen durch: Schlechtendalia chinensis Jacob Bell erzeugt werden. Dass die Pflanze, auf der sie wachsen, ebenfalls Rhus semialata M., habe ich schon nachzuweisen versucht: Es würden demnach die japanischen Gallen als besondere Art zu streichen sein, trotzdem aber als Handelssorte noch immer aufrecht erhalten werden müssen, denn die, wenn auch geringen Unterschiede lassen sich nicht wegleugnen.

Ueber die Art und Weise, wie diese Gallen sich öffnen, um schliesslich die Insekten zu entlassen, kann ich folgende Angaben machen: Man findet an manchen Stücken auf der Aussenfläche glasartig erhärtete Gerbsäure, unter diesen Tropfen finden sich oft Löcher in der Gallenwand, die nicht selten diese völlig durchbohren. Diese Löcher kommen hin und wieder in solcher Menge vor, dass die Gallen an solchen Stellen siebartig durchlöchert erscheinen.

Unter dem Mikroskop erkennt man mit Leichtigkeit auf Querschnitten, dass diese Zerstörung unter Braunfärbung des Zellinhalts dicht unter der Epidermis beginnt und sich allmählich im Innern fortsetzt. Nach Auflösung der Gerbsäure bleiben in den Zellen, die diesen corrodirten Stellen zunächst liegen, rundliche braune Klumpen zurück. -

Es liegt ja eigentlich der Gedanke nahe, anzunehmen, dass die Gallen durch Einreissung, ähnlich wie die nahe verwandten Ulmus Gallen sich öffnen, aber unter einer grossen Menge habe ich nicht ein einziges derartiges Stück auffinden können, es erscheint mir daher wahrscheinlich, dass die besprochenen Löcher zur Entlassung der geflügelten Insekten dienen. - 
Ferner habe ich unter den GalFig. 1. len meiner Sammlung 2 von $a b-$ weichender Beschaffenheit gefunden, die ich besprechen möchte:

1) In mehreren Stücken fand sich eine kleine Galle, von der ein vollständiges Exemplar etwa $1 \mathrm{Ctm}$. lang war, sich in zwei Aeste theilte, deren jeder wieder in zwei Spitzchen auslief, die Farbe ist gelbbraun, stellenweise lebhaft roth, die Wände papierdünn. (Fig. 1.) Mikroskopische Untersuchung: Auf die aus wenig plattgedrückten Zellen mit sehr vereinzelten Haaren bestehende Epidermis folgt ein Parenchym aus verhältnissmässig grossen Zellen. Etwa an der Grenze des zweiten Drittels des Querschnitts der Galle wird dies Parenchym von einer einfachen selten doppelten Schicht kleinerer, durchschnittlich etwas tangential

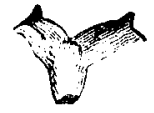

Fig. 2.

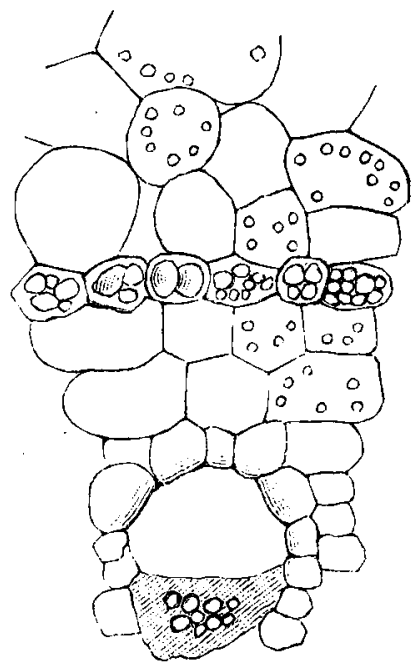
gestreckter Zellen unterbrochen, die mit Stärkekörnern dicht angefüllt sind. Einzelne Stärkekörner finden sich auch in den übrigen Zellen des Parenchyms. Nach der Innengrenze folgt dann ein einfacher Ring von Milchsaftschläuchen und darunter befindlichen Gefässbündeln, die Spiralgefässe enthalten. Das Parenchym zwischen diesen Milchsaftschläuchen und Gefässbündeln ist viel kleinzelliger als das übrige. (Fig. 2.)

Der Bau dieser Galle zeigt, dass wir es höchstwahrscheinlich ebenfalls mit einer Rhusgalle zu thun haben, doch ist sie den andern (japanischen und chinesischen) Rhusgallen gegenüber charakterisirt: 1) durch die sehr spärlich vorkommenden Haare, 2) die das Parenchym in zwei Hälften trennende Schicht kleinerer, dicht mit Amylum gefüllter Zellen, 3) die Eigenthümlichkeit, dass die Milchsaftschläuche und Gefässbündel nur in einfacher Schicht und zwar nahe der Innengrenze der Galle auftreten, 4) die ausserordentliche Dünnheit der Gallenwand. 5) Ob die dichotome Theilung der Galle Regel ist, kann nur an einer grösseren Menge derselben entschieden werden.

Arch. A. Pharm. XXII. Bds, 23. Hft. 
Fig. 4.

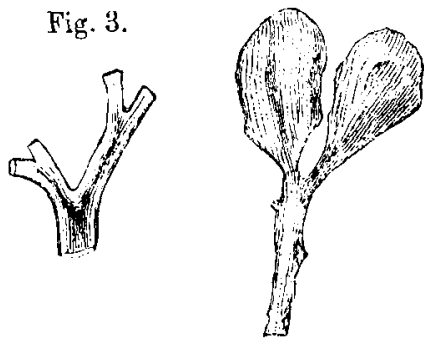

Fig. 5 .

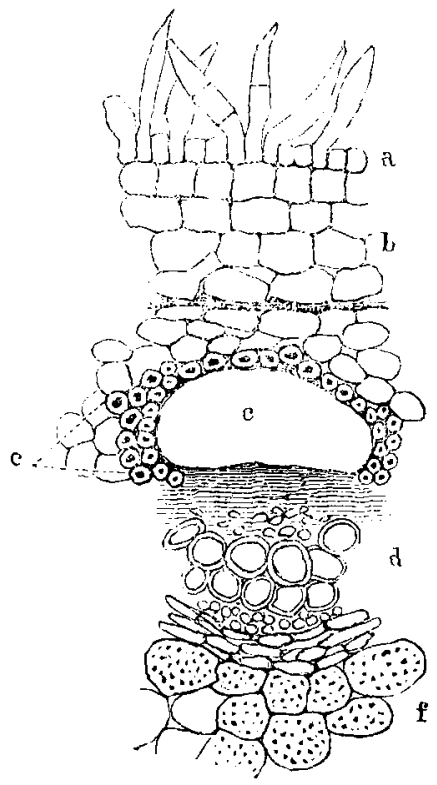

Den abweichend gebauten Ring stärkeführender Zellen möchte ich den Pleromscheiden vergleichen. (Vergl. De Bary, vergleich. Anatomie. pag. 431.) Dass eine solche Scheide von den Gefässbündeln durch eine wenigschichtige Parenchymzone getrennt ist, kommt ofter vor. -

2) Flückiger führt an, die chinesischen Gallen (also auch die japanischen) entatänden an $Z$ weigen. Ich glaube in meiner Sammlung zwei Stücke zu besitzen, bei denen der untere Theil aus dem veränderten Gewebe eines Zweiges besteht: Dieser untere Theil ist stengelförmig, nicht hohl; während der obere bei dem einen Stück aus zwei blasenförmigen Gallen gewöhnlicher Form besteht, ist er bei dem andern abgebrochen. (Fig. 3 u. 4.)

Mikroskopische Untersuchung (Fig. 5.) Auf die mit reichlichen Haaren besetzte Epidermis (a) folgt ein Parenchym, (b) in dem wie bei den gewöhnlichen japanischen Gallen die Milchsaftschläuche, (c) doch nur in einfacher Reihe verlaufen und unter ihnen starke Gefässbündel. (d) Auf der Aussenseite dieser Milchsaftschläuche finden sich häufig Gruppen sclerotischer Zellen, die dieselben gegen das Parenchym der Aussenrinde abgrenzen. (e) Diese sclerotischen Zellen finden sich in der Rinde von Rhus semialata ganz allgemein (De Bary, Vergleichende Anatomie pag. 466), bilden im unveränderten Zweig aber meist einen zusammenhänden Ring, während sie im vorliegenden Fall in einzelne Bündel aufgelöst sind. Für die Entstehung aus einem Zweig spricht ferner das Vorhandensein von Mark, (f) doch finden sich in diesem keine Milchsaftschläuche, die sonst für viele Rhus-Arten charakteristisch sind. 
Diese Gallen unterscheiden sich also von den gewöhnlichen : durch das Vorhandensein der Sklerenchym, die einfache Reihe von Milchsaftschläuchen und Gefässbündeln und das Vorhandensein von Mark; den Zweigen ähneln sie durch diese selben Merkmale, unterscheiden sich aber von ihnen dadurch, dass der Sklerenchymring in einzelne Bündel aufgelöst ist und die Milchsaftschläuche im Marke fehlen; doch ist nicht ausgeschlossen, dass diese im Marke ganz junger Zweige noch fehlen. -

Die beiden blasenförmigen Gallen an der Spitze des einen Exemplars lassen vermuthen, dass sie nicht nur aus einzelnen Blättern, sondern aus einer ganzen Knospe entstanden sind; als Analogon kann ich erwähnen, dass ich die Galle von Schizoneura lanuginosa, die nach Kessler (Lebensgeschichte der auf Ulmus campestris L. vorkommenden Aphiden etc. Cassel 1878) sich stets aus einer ganzen Knospe bilden soll, auch aus einem einzelnen Blatt entstanden gefunden habe.

\section{Deutscher Apothekerverein. Pharmakopöe-Com- mission.}

Eingesandt von Dr. G. Vulpius.

Entsprechend der übernommenen Aufgabe, von Zeit zu Zeit in der Vereinszeitschrift kurze Beschreibungen der Eigenschaften und Prüfungsweisen für neu in Gebrauch gekommene Arzneimittel in einer Form zu veröffentlichen, welche sich der von der Pharmakopöe gewählten möglichst anschliesst, beginnt unsere Commission heute mit den nachfolgenden 4 Artikeln.

Die betreffenden ersten Entwürfe, welche in ihrer unfertigen Gestalt durch einen Irrthum verfrüht in die pharmaceutische Tagespresse gelangten, sind nach brieflichem Verkehr mit den Fabrikanten provisorisch festgestellt und dann ausser den Commissionsmitgliedern noch verschiedenen Klinikern und Pharmakologen zur kritischen Prüfung unterbreitet worden, wobei eine Reihe yon Verbesserungsvorschlägen gemacht wurde, als deren schliessliches Ergebniss die nachstehenden Fassungen erscheinen. Ohne Anspruch auf Vollkommenheit zu machen, dürften sie als vorläufige Anhaltspunkte bis zur etwaigen späteren Aufnahme dieser Mittel in die Pharmakopöe sich nützlich erweisen. 